\title{
Embolização de pseudoaneurisma de artéria gastroduodenal em paciente com tuberculose extrapulmonar: relato de caso
}

\author{
Embolization of pseudoaneurysm of gastroduodenal artery in a patient with extrapulmonary \\ tuberculosis: case report
}

\author{
Embolización del pseudoaneurisma de la arteria gastroduodenal en un paciente com \\ tuberculosis extrapulmonar: reporte de un caso
}

Hyroan Brandell Pereira Corrêa ${ }^{1 *}$, Orlando José dos Santos ${ }^{1}$, Bruce Bezerra Martins ${ }^{1}$, Raimundo Teixeira de Araújo Júnior ${ }^{1}$, Thiago Sousa Dourado ${ }^{1}$, Vitória Gonçalves Alves de Oliveira ${ }^{1}$, Felipe Castelo Branco Rocha Silva1', Camila Judith Sousa San Lucas².

\section{RESUMO}

Objetivo: Descrever a evolução e o desfecho de um caso de pseudoaneurisma de artéria gastroduodenal em uma paciente com tuberculose extrapulmonar. Detalhamento do caso: Paciente do sexo feminino, 15 anos, sem comorbidades prévias conhecidas, deu entrada ao pronto-socorro com hematêmese sem controle por meio endoscópico e por esse motivo encaminhada ao centro cirúrgico para laparotomia de emergência. Durante cirurgia foi evidenciado tumoração em mesentério (diagnosticado posteriormente com tuberculose) e realizado duodenotomia e rafia de vaso com sangramento ativo. Topografia de lesão em segunda porção de duodeno. Evoluiu com novos episódios de hemorragia digestiva alta em pós-operatório, sendo diagnosticada com pseudoaneurisma de artéria gastroduodenal, realizando embolização com evolução favorável e alta com recuperação completa do quadro. Considerações finais: Pseudoaneurismas, normalmente, possuem uma origem secundária: traumática, inflamatória ou infecciosa. Pseudoaneurismas de artéria gastroduodenal são condições raras correspondendo a apenas $1,5 \%$ de todos os aneurismas viscerais. Na maioria das vezes, ele provoca sintomas inespecíficos, como dor abdominal, em presença ou ausência de ruptura, e hemorragia digestiva, retroperitoneal ou intraperitoneal. Embora o baixo número de casos conhecidos limite a experiência na abordagem terapêutica, normalmente se prefere o uso dos métodos endovasculares para abordagem dos mesmos.

Palavras-chave: Hemorragia gastrointestinal, Falso aneurisma, Tuberculose, Embolização terapêutica.

\begin{abstract}
Objective: To describe the evolution and outcome of a case of gastroduodenal artery pseudoaneurysm in a patient with extrapulmonary tuberculosis. Case details: Female patient, 15 years old, with no previous complications, was admitted to the emergency room with hematemesis without endoscopic control and for that reason was referred to the operating room for emergency laparotomy. During the surgery, tumor in the mesentery (later diagnosed with tuberculosis) and duodenotomy and raffia of the blood vessel were evidenced. Lesion in the second part of the duodenum. Evolved with new episodes of upper gastrointestinal bleeding in the postoperative period being diagnosed with pseudoaneurysm of gastroduodenal artery, performing artery embolization with evolution and discharge with complete recovery of the condition. Final considerations: Pseudoaneurysms usually have a secondary origin: traumatic, inflammatory or infectious. Gastroduodenal artery pseudoaneurysms are rare, corresponding to only $1.5 \%$ of all visceral aneurysms. Most of the time, it causes non-specific symptoms such as abdominal pain, in the presence or absence of rupture, and digestive hemorrhage, retroperitoneal or intraperitoneal. Although the low number of known cases limits the experience in the therapeutic approach, it is usually preferable to use endovascular methods to approach them.
\end{abstract}

Keywords: Gastrointestinal hemorrhage, Aneurysm, false, Tuberculosis, Embolization, therapeutic.

${ }^{1}$ Universidade Federal do Maranhão (UFMA), São Luís - MA. *E-mail: hyroan@gmail.com

2Universidade (CEUMA), São Luís - MA.

SUBMETIDO EM: 10/2020 ｜ ACEITO EM: 11/2020 ｜ PUBLICADO EM: 11/2020

REAS/EJCH | Vol.12(11) | e5637 | DOI: https://doi.org/10.25248/reas.e5637.2020 Página 1 de 6 


\section{RESUMEN}

Objetivo: Describir la evolución y el resultado de un caso de pseudoaneurisma de la artéria gastroduodenal en un paciente con tuberculosis extrapulmonar. Detalles del caso: La paciente, de 15 años de edad, sin complicaciones previas, ingresó a la sala de emergencias con hematemesis sin control endoscópico y, por esa razón, fue derivada al quirófano por laparotomía por enfermedad. Durante la cirugía, se evidenciaron el tumor en el mesenterio (luego diagnosticado con tuberculosis) y la duodenotomía y la rafia del vaso sanguíneo. Lesión en 1 parte del duodeno. Evolucionó con nuevos episodios de hemorragia digestiva alta en el postoperatorio diagnosticados con pseudoaneurisma de la arteria gastroduodenal, realizando embolización de la arteria con evolución económica y descarga con recuperación completa de la afección. Consideraciones finales: Los pseudoaneurismas suelen tener un origen secundaria: como traumática, inflamatória o infecciosa. Los pseudoaneurismas de la arteria gastroduodenal son poco frecuentes y corresponden solo al 1,5\% de todos los aneurismas viscerales. En la mayoría de los casos, causa síntomas inespecíficos, como dolor abdominal, en presencia o ausencia de ruptura, y hemorragia digestiva, retroperitoneal o intraperitoneal. Aunque el bajo número de casos conocidos limita la experiencia en el enfoque terapéutico, generalmente es preferible utilizar métodos endovasculares para abordarlos.

Palabras clave: Hemorragia gastrointestinal, Aneurisma falso, Tuberculosis, Embolización terapéutica.

\section{INTRODUÇÃO}

Aneurismas de artérias viscerais são pouco comuns, com incidência abaixo de $0,8 \%$, sendo os pseudoaneurismas de ocorrência ainda menor. Pseudoaneurismas representam aumentos focais do lúmen de um vaso decorrente de disrupção parcial ou total da vasculatura. Surgem mais comumente em decorrência de desordens inflamatórias, como pancreatite crônica, podendo ter etiologias menos frequentes, como aterosclerose, doenças do tecido conjuntivo, lesões traumáticas ou iatrogênicas, e até mesmo uma rara e temida complicação da tuberculose (SEITH A, et al., 2003; THAKUR KS, et al., 2011; PRETTE PR, et al., 2018).

A tuberculose (TB) é uma das doenças mais prevalentes do mundo e representa um sério e grave problema de saúde pública global. Nesse contexto, o Brasil apresenta-se como um dos países com maior número de casos. A TB pode ser definida de duas formas, a TB pulmonar, forma mais comum, quando afeta apenas parênquima pulmonar ou TB extrapulmonar quando afeta outras partes do corpo como meninges, rins, ossos, linfonodos, dentre outros (MASSABNI AC, et al., 2019).

A tuberculose abdominal é uma forma comum de tuberculose extrapulmonar e muitas vezes segue um curso insidioso com falta de sintomas específicos e achados radiográficos, o que gera um desafio constante no cuidado desses pacientes, podendo resultar em atraso no diagnóstico e tratamento, além de ter interferência direta no desfecho (PAULA C, 2019).

Os pseudoaneurismas já foram descritos anteriormente como complicação da tuberculose, na aorta femoral e em poucos casos envolvendo artérias viscerais como mesentérica e celíaca. Entretanto, em artéria gastroduodenal foi encontrado apenas um relato que apresentou hematêmese grave devido hemobilia e foi tratado de forma satisfatória por embolização (COELHO AP, et al., 2019).

Pseudoaneurismas de artéria gastroduodenal são incomuns e compreendem apenas a 1,5\% dos aneurismas viscerais. São ainda, em sua grande maioria, assintomáticos e diagnosticados de forma incidental, mas podem apresentar taxa de mortalidade de até 40 \% quando se manifestam com sinais e sintomas relacionados a sua ruptura, como hemoperitônio, hemorragia retroperitoneal ou gastrointestinal (NICHOLAS H, et al., 2013; BINETTI M, et al., 2019).

Portanto, o objetivo do estudo relatar um caso de pseudoaneurisma de artéria gastroduodenal em uma paciente com tuberculose extrapulmonar que deu entrada a um hospital de emergência do Maranhão com quadro de hemorragia digestiva alta, sendo tratada de forma minimamente invasiva pelo serviço de hemodinâmica.

REAS/EJCH | Vol.12(11) | e5637 | DOI: https://doi.org/10.25248/reas.e5637.2020 Página 2 de 6 


\section{DETALHAMENTO DO CASO}

Paciente do sexo feminino, 15 anos, com história de internação em um Hospital Universitário do Maranhão, há cerca de seis meses, por conta de quadro de hemorragia digestiva alta, sem identificação da causa do sangramento. Recebeu alta sem diagnóstico específico após realização de endoscopia digestiva alta (EDA), com laudo evidenciando apenas a presença de duodenite crônica e tomografia de tórax com achados inespecíficos, podendo corresponder a tuberculose, recebendo encaminhamento para ambulatório de pneumologia do serviço. O acompanhamento acabou não sendo realizado por problemas sociais.

A paciente foi admitida no serviço de emergência, acompanhada pela mãe, encaminhada do interior do estado, devido a novo quadro de hematêmese e melena. Ao exame físico, apresentava-se em regular estado geral, eupneica em ar ambiente, taquicárdica, com mucosas hipocoradas, abdome flácido, doloroso à palpação profunda principalmente em epigastro, mas sem sinais de irritação peritoneal. A mãe relatou história de febre vespertina há cerca de um mês mas negou doença hepática prévia, história de úlcera gástrica, sudorese noturna e tosse nesse período. Foi iniciada expansão volêmica, procedimentos de suporte e a paciente foi encaminhada para realização de endoscopia após estabilização. Durante o procedimento foi evidenciado sangramento volumoso próximo ao bulbo duodenal, mas sem possibilidade de controle por via endoscópica. A paciente evoluiu com instabilidade hemodinâmica e por esse motivo foi encaminhada ao centro cirúrgico para realização de laparotomia exploratória.

No intraoperatório foi encontrada uma tumoração em mesentério que dificultava a identificação dos marcos anatômicos, sendo realizada a biópsia para a pesquisa de bacilo álcool-ácido resistente (BAAR) e para o histopatológico. Foi realizada duodenotomia, rafia de vaso com sangramento ativo em segunda porção duodenal e passagem de sonda nasoentérica após a incisão com o objetivo de alimentar de forma precoce. Apesar da pesquisa de BAAR pela coloração Fite-Fáraco ter sido negativa, o nódulo mesentérico apresentava reação granulomatosa com necrose caseosa central, sugestivo de tuberculose. Pelo aspecto da lesão, idade e epidemiologia da região, seguiu-se protocolo de tratamento para tuberculose, com o esquema rifampicina, isoniazida, pirazinamida e etambutol (RIPE), sob orientação do serviço de infectologia do Hospital Universitário Presidente Dutra. Além disso foi realizado teste de HIV com resultado negativo.

Durante o pós-operatório a paciente teve boa evolução, com melhora dos níveis hematimétricos, com suporte alimentar por sonda nasoentérica, sem sinais de distensão abdominal ou êmese até o décimo dia, quando voltou a apresentar hemorragia digestiva, com hematêmese, melena, queda da hemoglobina para 4,5 g/dL e piora considerável do estado geral. Deu entrada em unidade de terapia intensiva no Hospital de urgência, onde foi iniciado suporte hemodinâmico, incluindo a transfusão de hemoconcentrados, sendo a paciente encaminhada novamente para a endoscopia, na qual foi levantada a suspeita de pseudoaneurisma da artéria gastroduodenal, que se encontrava sem sangramento ativo no momento.

A paciente foi transferida para a unidade de terapia intensiva de um Hospital Universitário que dispunha serviço de hemodinâmica. Durante admissão, foi realizada angiotomografia, que não revelou pseudoaneurisma. Apesar das medidas de estabilização, a paciente continuou com quadro de hemorragia digestiva importante e, por esse motivo, foi encaminhada para o serviço de hemodinâmica, no qual foi realizado exame arteriográfico via punção retrógrada de artéria femoral comum direita (Figura 1), evidenciando um pequeno pseudoaneurisma próximo à origem da artéria gastroduodenal, com interrupção da mesma antes da origem de seus ramos. Foi realizado cateterismo de tronco celíaco com cateter MIK 5 FR e superseletivação da artéria gastroduodenal com microcateter tipo PROGREAT e embolização com molas em todo o trajeto do pseudoaneurisma.

A arteriografia de controle (Figura 2) revelou tratamento adequado da lesão, com exclusão da artéria gastroduodenal. A paciente evoluiu com melhora do estado geral e dos padrões hematimétricos, sem novos episódios de sangramento ou outras intercorrências, recebendo alta, apenas dando continuidade ao esquema RIPE para tratamento da tuberculose.

REAS/EJCH | Vol.12(11) | e5637 | DOI: https://doi.org/10.25248/reas.e5637.2020 Página 3 de 6 
Figura 1 - Arteriografia via punção retrógrada de artéria femoral comum direita demonstrando pseudoaneurisma próximo à origem da artéria gastroduodenal.

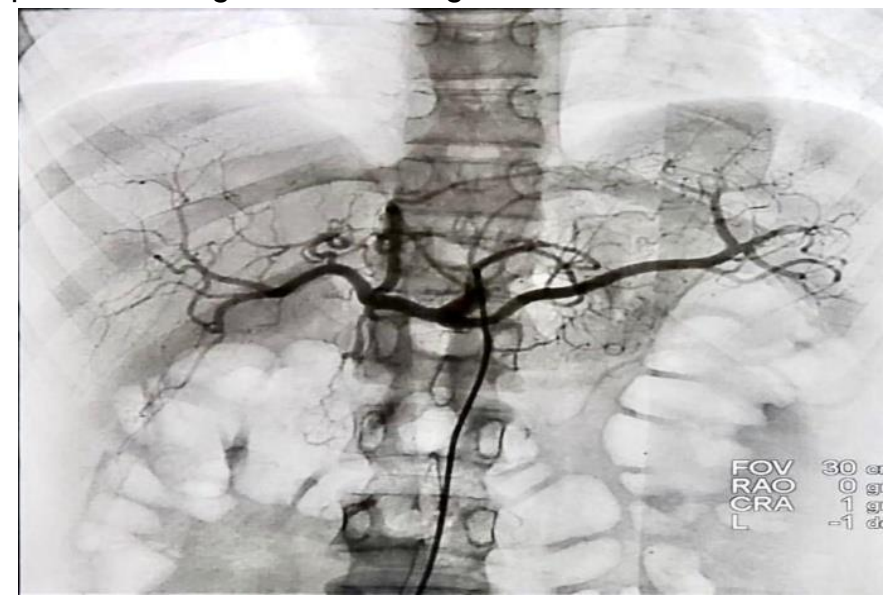

Fonte: CORREA HB, et al., 2020.

Figura 2 - Arteriografia de controle revelando tratamento adequado da lesão

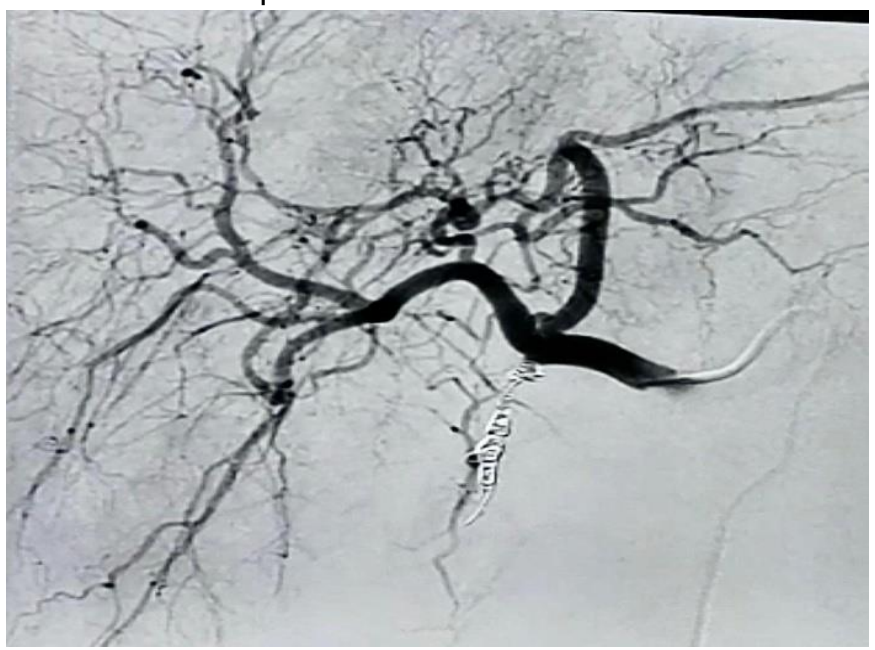

Fonte: CORREA HB, et al., 2020.

\section{DISCUSSÃO}

Os pseudoaneurismas de artéria gastroduodenal (GDA) são em sua maioria assintomáticos e diagnosticados de forma incidental em exames de imagem, no entanto, podem apresentar-se como uma situação clínica grave e potencialmente fatal (KOHAMA K, et al., 2016). No caso estudado, a paciente apresentou um quadro gravíssimo, que dada a história e associação aos achados clínicos apresentava sinais de ruptura que poderiam indicar uma mortalidade de até $40 \%$. Em relação a sua etiologia, a paciente apresentava 2 possíveis causas: a tuberculose ou causa iatrogênica, pela cirurgia de urgência realizada antes do diagnóstico. Causas incomuns para uma afecção rara, tornando a descrição aqui relatada uma informação científica consistente no manejo dessa enfermidade.

Quando relacionado à tuberculose descrevem-se duas possíveis vias de disseminação para parede arterial: a primeira seria extensão direta de lesões contíguas, como linfonodos infectados, empiema ou pericardite e a 
segunda disseminação hematogênica ou linfangítica de lesões primárias. Pseudoaneurismas tuberculosos foram relatados no arco aórtico distal e porção supradiafragmática da aorta torácica descendente, devido à proximidade de linfonodos infectados. No abdome, os nódulos tuberculosos mais comumente envolvidos são os grupos portal e peripancreático. O envolvimento do GDAno paciente poderia ser explicado pela extensão contígua dos nódulos necróticos adjacentes (OHTSUKA T, et al., 1996; GULATI MS, et al., 1996).

Em relação ao diagnóstico do pseudoaneurisma, este pode ser realizado por métodos de imagem não invasivos como angiotomografia, com elevada sensibilidade, além da ecografia e angiorressonância magnética. O método gold standard é a angiografia que tem potencial diagnóstico e terapêutico (SANKET T, et al., 2015). No presente relato, a angiotomografia não foi realizada, o que poderia retardar o diagnóstico e ter interferência significativa no desfecho do caso. Mas tendo em vista a alta suspeita diagnóstica e o quadro de hematêmese e melena foi realizado a angiografia, que nesse contexto, mostra-se fundamental, pois fornece informações importantes sobre a anatomia vascular (dimensão e tamanho do colo), além de apresentar um potencial terapêutico (NEJMEDDINE A, et al., 2008).

Em relação ao diagnóstico de TB extrapulmonar no caso foi realizado por meio do critério clínico epidemiológico como aspecto da lesão, idade, sexo e epidemiologia da região mesmo com a pesquisa para BAAR pelo método Fite-Fáraco sendo negativa, já que isso pode ser explicado pelo número de bacilos presentes nesses tecidos que são muitas vezes baixos, além da paciente apresentar outros fatores de risco para TBEP como: ser menor de idade, sexo feminino e raça não-branca, fatores de risco podem diferir daqueles presentes na TBP (LAWN SD, et al., 2012; YANG Z, et al., 2004; PETO HM, et al., 2009; FISKE CT, et al., 2010). Outro importante fator de risco é infecção pelo HIV, mas como descrito anteriormente o teste foi negativo.

O tratamento do pseudoaneurisma de artéria gastroduodenal está preconizado independente da dimensão, mas a escolha do procedimento deve ser individualizada (NICHOLAS H, et al., 2013). As estratégias terapêuticas incluem procedimentos cirúrgicos ou endovasculares. Estes últimos constituem atualmente o tratamento de escolha nos casos detectados incidentalmente, sem rotura, e nas situações de rotura, desde que hemodinamicamente estáveis (RYAN Y, et al., 2004).

Os procedimentos endovasculares apresentam vantagens por serem menos invasivos e diminuírem o risco de complicações; têm como objetivo a exclusão do pseudoaneurisma da circulação, podendo ser realizado de duas maneiras: embolização e/ou colocação de stents. Os stents revestidos ou não-revestidos podem ser utilizados em quase todos os casos, mas, principalmente, quando se deseja manter a perviedade da artéria, e requerem condições anatômicas favoráveis para seu emprego; a artéria de origem do pseudoaneurisma deve ser acessível, ter um calibre adequado, sem significativas tortuosidades (NASSER F, et al., 2010).

Em relação à embolização, destacam-se duas abordagens: embolização do saco do pseudoaneurisma com preservação da vascularização adjacente - reservada a pseudoaneurismas com origem em territórios vasculares críticos que não permitem a oclusão do vaso de origem - ou, alternativamente, oclusão do vaso que origina o pseudoaneurisma, distalmente e proximalmente em relação ao colo, utilizada em pseudoaneurismas com origem em territórios vasculares não críticos (DUTRA S, et al.,2017).

O tratamento no caso foi realizado de forma minimamente invasiva, como recomenda a literatura, por meio cateterismo de tronco celíaco com cateter MIK 5 FR e superseletivação da artéria gastroduodenal com microcateter tipo PROGREAT e embolização em todo o trajeto do pseudoaneurisma com molas, que são dispositivos metálicos destinados a causar trombose permanente dos vasos, tal como demonstrado na arteriografia de controle após a embolização. A vascularização colateral prévia entre as artérias viscerais permite a exclusão ou a não reconstrução vascular da artéria gastroduodenal nos casos de embolização ou ressecção, respectivamente, a menos que haja oclusão da artéria celíaca. Nesse caso para aqueles pacientes com exclusão da artéria celíaca ou estenose da artéria mesentérica superior, angioplastia transluminal seria forma de diminuir o risco de complicações isquêmicas como como gangrena de vesícula biliar e estômago, necrose esplênica ou 
outras consequências desastrosas. Por isso é essencial a individualização dos casos e da seleção adequada da técnica e do material (NICHOLAS H, et al., 2013; SARIOGLU O, et al., 2019).

Em condições ideais, o fluxo colateral adequado deve ser documentado com imagens pré-operatórias, se permitido. Dois exames não invasivos demonstraram excelente resolução para planejamento pré-operatório: a angiotomografia e a angioRNM. Entretanto, a arteriografia também pode ser realizada, como no caso em questão, principalmente por ter sido realizada em caráter de urgência e permitir avaliação do fluxo em tempo real e de forma dinâmica (CASAS ALF, et al., 2019).

O pseudoaneurisma de artéria gastroduodenal é raro, mas pode resultar em situações catastróficas, com complicações de potencial fatalidade. O tratamento endovascular mediante embolização arterial é menos invasivo e apresenta menor morbimortalidade quando comparado à cirurgia convencional, sendo a terapêutica de escolha nos casos em que não há rotura e em casos de rotura em pacientes hemodinamicamente estáveis. Quando realizado em um centro de referência e por profissionais especializados, os benefícios destes procedimentos suplantam as complicações, apresentando uma taxa de sucesso entre $70 \%$ e $100 \%$. A radiologia de intervenção desempenha um papel al no tratamento desta patologia, além de fornecer parâmetros de acompanhamento para uma evolução favorável.

\section{REFERÊNCIAS}

1. BAVUNOGLU I, et al. Selective Jejunal Artery Pseudoaneurysm Embolization In A Patient With Massive Gastrointestinal Bleeding Due To Intestinal Tuberculosis. The Journal of Emergency Medicine 2006; 31(4): 391-394.

2. BBINETTI M, et al. Pseudoaneurisma da artéria gastroduodenal em um paciente com sangramento recorrente: relato de caso e revisão da literatura. Doenças e Ciências Digestivas 2019; 64 (11): 3086-3091.

3. CASAS ALF, et al. Tratamento endovascular de aneurisma de artéria gastroduodenal: relato de caso. Jornal Vascular Brasileiro 2019; 18: $1-5$

4. COELHO AP, et al. Infectious celiac artery aneurysm —a rare clinical entity. Angiologia e Cirurgia Vascular 2019; 15(1): 19-24.

5. CORBY R, et al. Mesenteric pseudoaneurysm in a 12-year-old girl with abdominal tuberculosis presenting with massive lower gastrointestinal bleeding. Pediatr Radiol 2006; 36: 456-459.

6. DUTRA S, et al. Endovascular Treatment of a Gastroduodenal Artery Pseudoaneurysm Rupture after a Car Accident. Acta Med Port 2017; 30(9): 658-661.

7. FISKE CT, et al. "Black race, sex, and extrapulmonary tuberculosis risk: an observational study." BMC Infect Dis 2010; 10-16.

8. GULATI MS, et al. CT appearances in abdominal tuberculosis - a pictorial essay. Clin Imaging 1999; 23:51-9.

9. KIM JK, et al. Hematemesis Due to a Pseudoaneurysm of the Splenic Artery Secondary to Gastric Tuberculosis. Cardiovasc Intervent Radiol 2005; 28: 506-508

10. KOHAMA K, et al. Successfully treated life-threatening upper gastrointestinal bleeding from fistula between gastroduodenal artery pseudoaneurysm and duodenum. Acute Medicine \& Surgery 2016; 3: 192-194.

11. LAWN SD, et al. Diagnosis of extrapulmonary tuberculosis using the Xpert((R)) MTB/RIF assay. Expert Rev Anti Infect Ther 2012; 10(6): 631-635.

12. MASSABNI AC, et al. Tuberculose: história e evolução dos tratamentos da doença. Revista Brasileira Multidisciplinar $2019 ; 22$ (2): 6-34.

13. NASSER F, et al. Endovascular treatment for superior mesenteric artery pseudoaneurysm: case report. Vasc Bras 2010; 9 (3): $182-185$.

14. NICHOLAS H, et al. Gastroduodenal artery aneurysm, diagnosis, clinical presentation and management: a concise review. Annals of Surgical Innovation and Research 2013, 7(4): 1-6.

15. OHTSUKA T, et al. Tuberculous pseudoaneurysm of thoracic aorta. Ann Thorac Surg 1996; 62: $1831-4$.

16. ORAN I, et al. Mesenteric artery aneurysms in intestinal tuberculosis as a cause of lower gastrointestinal bleeding. Abdom Imaging 2001; 26: $131-133$.

17. PAULA C. Tuberculose - manifestação gastrointestinal: Relato de caso e revisão da literatura. Trabalho de conclusão de curso (Graduação em Medicina). UniCEUB, Brasilia, 2019, 34p.

18. PETO HM, et al. Epidemiology of extrapulmonary tuberculosis in the United States, 1993-2006. Clin Infect Dis 2009; $49(9): 1350-1357$.

19. PRETTE PR, et al. Endovascular treatment of acute gastrointestinal bleeding from a large splenic artery pseudoaneurysm: case report and literature review. Jornal vascular brasileiro, 2018; 17(3), 234

20. RYAN Y, et al. Gastroduodenal Artery Pseudoaneurysm Secondary to Pancreatic Head Biopsy. J Ultrasound Med 2004; $23: 997-1001$.

21. SANKET T, et al. Obscure gastrointestinal bleeding secondary to gastroduodenal pseudoaneurysm. The Southwest Respiratory and Critical Care Chronicles 2015; 3(12).

22. SARIOGLU O, et al. Opções de tratamento intervencionista em pseudoaneurismas: diferentes técnicas em diferentes localizações. Jornal Polonês de Radiologia 2019; 84: 319 - 327.

23. SEITH A, et al. Tuberculous pseudoaneurysm of gastroduodenal artery. Journal of Clinical Imaging 2003; 27: 408-410.

24. THAKUR KS. Gastro Duodenal Artery Aneurysm: An Uncommon Complication of Duodenal Ulcer. JournalofDigestiveEndoscopy 2011; 2 (2): $154-156$.

25. YANG Z, et al. Identification of risk factors for extrapulmonary tuberculosis. Clin Infect Dis 2004; 38(2): 199-205.

REAS/EJCH | Vol.12(11) | e5637 | DOI: https://doi.org/10.25248/reas.e5637.2020 Página 6 de 6 